\title{
Improving the productivity and efficiency of vascular surgery and radiology multidisciplinary meetings
}

Gary Dobson, Declan Neeson

Belfast Health \& Social Care Trust, UK

\begin{abstract}
Multidisciplinary team meetings are recognized as an important factor in driving quality of care. The Vascular Society of Great Britain and Northern Ireland recommend that all aspects of vascular surgery are suitable for discussion at multidisciplinary team (MDT) meetings, including carotid, peripheral, and aortic disease.
\end{abstract}

The juniors doctors were tasked with preparing the patient list in our unit. This had become time consuming and somewhat unrewarding. The methods of preparation and information required on the patient list were reviewed.

It was felt that typing a clinical summary for each patient on the list was the most time consuming factor. This was removed and patients were instead simply categorized into subgroups such as aneurysms, critical limb ischemia, and others.

The information removed was substituted with the use on an electronic care record during the meeting to answer questions regarding comorbidities and previous interventions.

Time spent preparing the meeting by the junior doctors was recorded before and after cycles of intervention. Prior to intervention this was found be a mean of 140 minutes ( 2.3 hours), and improved to a mean of 45 minutes ( 0.45 hours) with consequent cycles.

The overall proportion of patients receiving definitive outcomes in each meeting after changes were implemented increased from 35 to $55 \%$. This was not a primary outcome, but an unintended consequence of careful consideration of each cycle.

In conclusion, the changes implemented have allowed junior doctors to spend more of their time with other activities such as the outpatient department (OPD) and theatre during their vascular surgery rotation. The proportion of cases discussed has increased due to more focused discussions during the MDT meeting, resulting in improved patient care. All stakeholders gave a largely positive reaction to the changes implemented.

\section{Problem}

Preparation of the weekly regional vascular and radiology multidisciplinary team meeting in the Royal Victoria Hospital, Belfast, is the responsibility of the foundation year 2 (FY2) and core trainees 1/2 (CT1/2) trainees who work in the department. Those involved in its preparation found it to be a somewhat laborious process and took away time that could be spent on other clinically important tasks such as OPD and theatre.

The issue was discussed with the consultants within the department who agreed that change would be welcome. It was also felt that that the current methods employed did not necessarily provide the most relevant information for the consultants at the meeting, and therefore the lack of succinctness tended to slow the discussion of patients. There was also some frustration on behalf of the radiologists in regards to the same problem.

\section{Background}

The Vascular Society of Great Britain and Northern Ireland (VSGBI) recognize multi-disciplinary team working as a "key factor in driving quality of care provision".[1] Indeed, particularly for management of abdominal aortic aneurysms, the VSGBI framework for quality improvement specifies that all elective procedures should be reviewed preoperatively in a multi-disciplinary team that at least includes surgeons and radiologists.[2]

The method of preparing the MDT within our unit had been in place for a number of years, and was seen by trainees as a task to be avoided if at all possible. Despite this the meeting was dutifully prepared each week and no major attempts had been made to change the process.

As we had no evidence of previous attempts at change we proceeded carefully, and took opinions from as many stakeholders in the meeting as possible.

\section{Baseline measurement}

All foundation year 2 and core trainees involved in preparing the 
BMJ Quality Improvement Reports

meeting were retrospectively surveyed (six in total) and asked to estimate the amount of time they spent updating the patient list each week. The mean time was 140 minutes (range 120 to 150).

The number of cases put forward for discussion ranged from 61 to 94 over a two month period. The proportion of these discussed with outcomes documented had a mean of 22.25 cases, equivalent to $35 \%$ (range; 17 to 26 cases, or 26 to $38 \%$ ).

\section{Design}

During initial discussions it was felt that the most time consuming factor was writing a brief clinical summary from the case notes provided. The Northern Ireland electronic care record (NIECR) has complete copies of all outpatient clinic letters and inpatient discharges, along with most recent laboratory results and radiology reporting, but not images. This resource is available to all medical staff in Northern Ireland and accessible to the MDT during the meeting. It was possible to make use of a second projector screen so that all attendees could read the information available.

Therefore the initial intervention was to remove the clinical summary from the patient list, and use the online NIECR to answer any clinical queries within the meeting.

Before implementation the proposal was discussed with the consultants and secretarial staff as a trial design for one week.

\section{Strategy}

PDSA cycle 1: The clinical summary for each patient was removed from the typed list of patients. The electronic care record was used during the meeting to good effect. The new format of the patient list was reviewed following the MDT meeting. Secretarial staff found that they no longer had a clinical summary to type the MDT outcome. This was an oversight during design. This was corrected by consultants providing a summary referral letter for each patient to be discussed at the meeting. This letter was to be placed at the front of the case notes for each patient.

PDSA cycle 2: The changes implemented had reduced preparation time of the meeting for junior doctors, and the new referral process had provided succinct clinical information as required. The radiologists in the meeting however noted that the new list of patients did not provide an indication of where the radiology images were to be found. Our regional MDT had images supplied by three separate radiology systems, and it was necessary to add the correct system for each patient to direct the radiologists appropriately.

PDSA cycle 3: The final alteration to the patient list was a suggestion to provide column space for consultants to make notes in response to outcomes and follow up arrangements.

PDSA cycle 4: The new format for the patient list was finalised and well received by all stakeholders. Satisfaction levels were high, and post project measurements were taken.

\section{Results}

The foundation year 2 and core trainees preparing the meeting were asked to record the time they spent with this task. Mean time had improved to 45 minutes (range 30 to 60 minutes).

The number of cases put forward for discussion ranged from 45 to 78 over a four week period, but the proportion of these discussed with outcomes documented had improved to a mean of 35 cases, equivalent to $55 \%$ (range 25 to 47 cases, or 50 to $61 \%$ ). This represents increase of approximately two thirds in the proportion of cases discussed.

\section{Lessons and limitations}

The success of this improvement has been dependent on the engagement of all members of the team affected by proposed changes. All those involved responded positively and were willing to contribute ideas to help the project succeed.

IT has once again proved to be a key component in improving how systems work, but small sequential steps are better than major and untried changes. The limitations would be recent turnover in staff, and it will be interesting to revisit the work done here in a number of months to see if the improvement has been maintained, or indeed bettered. This will be crucial to assess the long term impact, and possibly guide further developments.

\section{Conclusion}

The changes made to the process have resulted in a real reduction in the time spent preparing each weekly meeting. As a result of careful and thoughtful improvement cycles, it has been possible to increase the proportion of patients discussed at each meeting by approximately two thirds. This was not one of the primary outcomes of the process, but was a very welcome positive change that will improve patient care on a regional level.

The project has identified other ways in which the weekly MDT can be improved to benefit patient care, and steps are being taken to make further changes wherever possible. It is interesting to note how by undertaking this process to improve the primary outcome we have in fact improved other areas of our practice, and found other areas of our practice that can also be improved.

\section{References}

1. The Vascular Society of Great Britain \& Ireland. The Provision of Services for Patients with Vascular Disease. 2014. www.vascularsociety.org.uk/2014-the-provision-ofservices-for-patients-with-vascular-disease/

2. The Vascular Society of Great Britain \& Ireland. Framework for improving the results of elective AAA repair. 2011.

\section{Declaration of interests}


None to declare.

\section{Acknowledgements}

Regional vascular unit, Royal Victoria Hospital Belfast. Mr L Lau, consultant vascular surgeon.

\section{Ethical approval}

This work has been deemed an improvement study, and as such did involve any interaction with patients. All data were entirely anonymised and did require the holding of individual patient data. 